\title{
A Bayesian Threshold-Normal Mixture Model for Analysis of a Continuous Mastitis-Related Trait
}

\author{
J. Ødegård, ${ }^{1}$ P. Madsen, ${ }^{2}$ D. Gianola, ${ }^{1,3}$ G. Klemetsdal, ${ }^{1}$ J. Jensen, ${ }^{2}$ \\ B. Heringstad, ${ }^{1}$ and I. R. Korsgaard ${ }^{2}$ \\ ${ }^{1}$ Department of Animal and Aquacultural Sciences, Norwegian University of Life Sciences, \\ $\mathrm{N}-1432$ Ås, Norway \\ ${ }^{2}$ Department of Genetics and Biotechnology, Danish Institute of Agricultural Sciences, \\ Research Centre Foulum, DK-8830 Tjele, Denmark \\ ${ }^{3}$ Department of Animal Sciences, University of Wisconsin-Madison, \\ Madison 53706
}

\section{ABSTRACT}

Mastitis is associated with elevated somatic cell count in milk, inducing a positive correlation between milk somatic cell score (SCS) and the absence or presence of the disease. In most countries, selection against mastitis has focused on selecting parents with genetic evaluations that have low SCS. Univariate or multivariate mixed linear models have been used for statistical description of SCS. However, an observation of SCS can be regarded as drawn from a 2- (or more) component mixture defined by the (usually) unknown health status of a cow at the test-day on which SCS is recorded. A hierarchical 2-component mixture model was developed, assuming that the health status affecting the recorded test-day SCS is completely specified by an underlying liability variable. Based on the observed SCS, inferences can be drawn about disease status and parameters of both SCS and liability to mastitis. The prior probability of putative mastitis was allowed to vary between subgroups (e.g., herds, families), by specifying fixed and random effects affecting both SCS and liability. Using simulation, it was found that a Bayesian model fitted to the data yielded parameter estimates close to their true values. The model provides selection criteria that are more appealing than selection for lower SCS. The proposed model can be extended to handle a wide range of problems related to genetic analyses of mixture traits.

(Key words: Bayesian methods, mastitis, mixture model, somatic cell score)

Abbreviation key: IM = standard univariate model for SCS ignoring the mixture, LNM = liability normal

Received November, 23, 2004

Accepted March 18, 2005.

Corresponding author: J. Ødegård; e-mail: jorgen.odegard@ umb.no. mixture, $\mathbf{N M}=$ mixture model ignoring the structure of the underlying liability to mastitis.

\section{INTRODUCTION}

Arguably, SCS in the milk of a cow can be viewed as a variable drawn from a mixture distribution with a finite number of components. Each component would represent SCS records on cows from different udder health classes (e.g., healthy, subclinical, or clinical mastitis). A realistic model must account for the fact that udder health status is not static, as it may change in the course of lactation. For example, the probability of clinical mastitis is higher early in lactation than at later stages (Heringstad et al., 1999). This implies that, at a given time, an observed SCS may stem from distributions corresponding to cows not yet exposed to mastitis, mastitic cows, or cows that have recovered from the disease. Further, mastitis could be divided into clinical and subclinical cases or according to type of pathogen associated with the IMI. For the purpose of this study, it is convenient (and simpler) to assume that health status on a specific test-day can be such that either the cow is mastitic (IMI+) or that IMI is absent (IMI-). The distribution of observed SCS would then be a mixture of 2 components with different means (affected cows have a larger value) and, possibly, different variances as well.

Conceptually, one may think of a "baseline" SCS level that is affected by systematic (e.g., herd-year-season, age at calving, stage of lactation) and random (e.g., genetic, permanent environment) sources of variation. Mastitis would produce a shift away from the "baseline" level peculiar to a given combination of explanatory factors. Thus, an observed test-day SCS can be regarded as resulting from effects of several possibly confounded factors, such as "baseline" SCS (a continuous trait) and of shifts caused by a binary process (IMI-, IMI+). Ødegård et al. (2003) and Gianola et al. (2004) described a mixture model for SCS containing fixed and random 
effects affecting "baseline" SCS, with mastitis producing a change (typically upward) in the mean of the distribution. Based on simulated data, Ødegård et al. (2003) found that when residual variances were homogeneous, the model yielded seemingly unbiased estimates of most parameters; when these variances were heterogeneous between healthy and diseased animals, some bias was detected. This bias was no longer detectable after a programming error was found and was corrected subsequent to publication. In Ødegård et al. (2003), it was assumed that the random variable assigning observations to putative IMI- and IMI+ classes was independently distributed as Bernoulli, having the same a priori probability for all animals and all observations (i.e., test-day scores) within animal. Subsequent analyses of clinical mastitis field data have indicated that systematic (e.g., herd-year-season, age at calving, stage of lactation) and random (e.g., genetic, permanent environment) effects affect the probability of mastitis infection (Chang et al., 2004). Hence, assigning the same Bernoulli distribution, i.e., the same prior probability of group membership, to all animals at all testdays is not realistic. Thus, factors affecting probability of group membership should be included in the model to arrive at an improved statistical description.

Our objective was to extend the model of Ødegård et al. (2003), where SCS is treated as a 2-component mixture, by introducing a hierarchical structure for the group membership variables. In particular, the probability of membership in the IMI+ class, e.g., is allowed to vary with "fixed" and random effects that are distinct from those affecting SCS. The behavior of the model and of the procedures used for inference was examined with simulated data. The proposed model can be used for prediction of breeding values for liability to mastitis derived from observed SCS.

\section{MATERIALS AND METHODS}

\section{General}

Setting and notation are as described in Ødegård et al. (2003). Briefly, the data consist of $n$ measurements for a quantitative trait, such as SCS or electrical conductivity measured in the milk of a cow. A 2-component Gaussian mixture model poses that measurement $i$ of SCS, $S C S_{i}$ ( $i=$ animal or record within animal), given some location and dispersion parameters $(\boldsymbol{\alpha})$, and probabilities $(\mathbf{P})$ has the mixture density:

$$
\begin{gathered}
S C S_{i} \mid \boldsymbol{\alpha}, \mathbf{P} \sim N\left(f_{i}(\boldsymbol{\alpha}), g_{i}(\boldsymbol{\alpha})\right) P_{i} \\
+N^{*}\left(f_{i}^{*}(\boldsymbol{\alpha}), g_{i}^{*}(\boldsymbol{\alpha})\right)\left(1-P_{i}\right)
\end{gathered}
$$

where $\mathbf{P}=\left[P_{1}, P_{2}, \ldots, P_{n}\right]^{\prime}, P_{i}$ is the a priori probability that $S C S_{i}$ is drawn from distribution $N(\cdot)$ (diseased), and $\left(1-P_{i}\right)$ is the a priori probability that $S C S_{i}$ is drawn from $N^{*}(\cdot)$ (healthy). Ødegård et al. (2003) assumed $P_{i}=P$, for all $i$; in this study, $P_{i}$ may differ between observations. Further, $f_{i}(\boldsymbol{\alpha}), g_{i}(\boldsymbol{\alpha}), f_{i}^{*}(\boldsymbol{\alpha})$, and $g_{i}^{*}(\boldsymbol{\alpha})$ are functions of the parameter vector $\boldsymbol{\alpha}$. Typically, $f_{i}(\boldsymbol{\alpha})$ and $f_{i}^{*}(\boldsymbol{\alpha})$ are linear combinations of fixed and random effects; $g_{i}(\boldsymbol{\alpha})=\sigma_{\mathrm{e}{ }_{S C S}}^{2}$ and $g_{i}^{*}(\boldsymbol{\alpha})=\sigma_{\mathrm{e} 0_{S C S}}^{2}$ for all $i=1,2$, $\ldots, n$, where $\sigma_{\mathrm{e} 0_{S C S}}^{2}$ and $\sigma_{\mathrm{e} 1_{S C S}}^{2}$ are variance parameters. Given $\boldsymbol{\alpha}$ and $\mathbf{P}$, observations were assumed to be conditionally independent, so that the joint density of the data vector SCS is

$$
\begin{gathered}
p(\mathbf{S C S} \mid \mathbf{P}, \boldsymbol{\alpha})=\prod_{i=1}^{n}\left[N\left(f_{i}(\boldsymbol{\alpha}), g_{i}(\boldsymbol{\alpha})\right) P_{i}\right. \\
\left.+N^{*}\left(f_{i}^{*}(\boldsymbol{\alpha}), g_{i}^{*}(\boldsymbol{\alpha})\right)\left(1-P_{i}\right)\right] .
\end{gathered}
$$

Estimation, either by maximum likelihood (Gianola et al., 2004) or by Bayesian (Ødegård et al., 2003) approaches, is facilitated by augmenting the density described above with auxiliary binary indicator (IMI $-\rightarrow$ 0 , IMI $+\rightarrow 1)$ variables $Z_{i}(i=1,2, \ldots, n)$. Assuming that the indicator variables are product Bernoulli and conditionally independent a priori, one can write

$$
\begin{aligned}
& \operatorname{Pr}(\mathbf{Z}=\mathbf{z} \mid \mathbf{P}, \boldsymbol{\alpha})=\operatorname{Pr}(\mathbf{Z}=\mathbf{z} \mid \mathbf{P}) \\
= & \prod_{i=1}^{n} \operatorname{Pr}\left(Z_{i}=z_{i} \mid P_{i}\right)=\prod_{i=1}^{n} P^{z_{i}}\left(1-P_{i}\right)^{1-z_{i}}
\end{aligned}
$$

where $\operatorname{Pr}\left(z_{i}=1 \mid P_{i}\right)=P_{i}$ is the prior probability that the health status of observation $i$ is IMI+, allowing for individual prior probabilities of mastitis, which is an extension of Gianola et al. (2004) and Ødegård et al. (2003).

In our "extended" mixture model, we postulate an underlying continuous random variable, called liability $(\lambda)$, which determines the mastitis status associated with each observation $\left(Z_{i}\right)$ depending on the value of liability relative to a fixed threshold. This is called a threshold-liability model (Wright, 1934; Dempster and Lerner, 1950; Falconer, 1965; Gianola, 1982; Gianola and Foulley, 1983), which has been used for genetic analysis of clinical mastitis as a binary response (e.g., Heringstad et al., 2003). Here, liability is incorporated into what we term as a liability-normal mixture (LNM) model. Both in LNM and in standard threshold models, it is assumed that mastitis status switches from 0 to 1 if liability exceeds a given threshold $T$. In the standard threshold model, data consist of observed binary responses (e.g., presence or absence of clinical mastitis), 
whereas, in the LNM model, data consist of observed SCS, which is a continuous valued variable. However, the true distribution of SCS switches from $N^{*}(\cdot)$ to $N(\cdot)$ according to mastitis status, and putative mastitis status may be inferred from the observed SCS. Thus, in the LNM model, the a priori probability of IMI+ for a specific SCS observation $i$ can be written as

$$
P_{i}=\operatorname{Pr}\left(\lambda_{i}>T \mid \alpha\right)
$$

so that

$$
Z_{i}=\left\{\begin{array}{l}
0 \text { if } \lambda_{i} \leq T \\
1 \text { if } \lambda_{i}>T
\end{array}\right.
$$

where $\lambda_{i}$ is the liability variate and $T$ is the threshold (we take $T=0$ ). Thus, in this model, $P_{i}$ depends on some of the elements in $\alpha$.

In the following, we argue that for this model, it is reasonable to assume that

$$
\begin{gathered}
\mathrm{p}(\boldsymbol{\lambda}, \mathbf{S C S} \mid \mathbf{Z}=\mathbf{z}, \boldsymbol{\alpha})= \\
\mathrm{p}(\boldsymbol{\lambda} \mid \mathbf{S C S}, \mathbf{Z}=\mathbf{z}, \boldsymbol{\alpha}) \mathrm{p}(\mathbf{S C S} \mid \mathbf{Z}=\mathbf{z}, \boldsymbol{\alpha})= \\
\mathrm{p}(\boldsymbol{\lambda} \mid \mathbf{Z}=\mathbf{z}, \boldsymbol{\alpha}) \mathrm{p}(\mathbf{S C S} \mid \mathbf{Z}=\mathbf{z}, \boldsymbol{\alpha}) .
\end{gathered}
$$

That is, given $\mathbf{Z}=\mathbf{z}$, liability is conditionally independent of SCS. In other words, given $\mathbf{Z}=\mathbf{z}$, which in turn is inferred from SCS, and $\alpha$, SCS does not convey any additional information about liability. This implies that the residual correlation between SCS and liability should be assumed to be zero.

\section{Modeling SCS and Liabilities}

Let

$$
\alpha=\left(\beta, \mathbf{a}, \mathbf{p}, \mathbf{G}_{0}, \mathbf{P}_{\mathbf{0}}, \sigma_{e 0_{\mathrm{SCS}}^{2}}, \sigma_{e 1_{\mathrm{SCS}}}^{2}\right)
$$

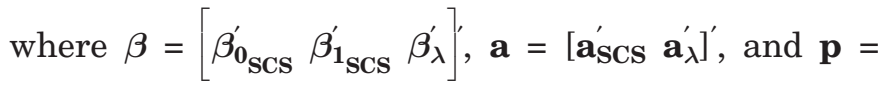

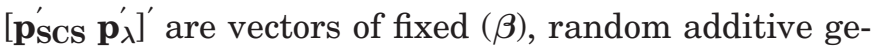
netic (a), and random permanent environmental (p) effects on SCS and liability to mastitis. Further, $\mathbf{G}_{\mathbf{0}}$ is the $2 \times 2$ additive genetic (co)variance matrix between SCS and $\lambda$, and $\mathbf{P}_{\mathbf{0}}$ is the $2 \times 2$ (co)variance matrix of permanent environmental effects; $\sigma_{\mathrm{e} 0}^{2}{ }_{\mathbf{S C S}}$ and $\sigma_{\mathrm{e} 1}^{2}{ }_{\mathbf{S C S}}$ are the residual variances of SCS in IMI- and IMI+ classes, respectively. Given disease status $(\mathbf{Z})$ and $\alpha$, SCS can be modeled as a standard Gaussian trait, allowing for heterogeneous residual variance for the different disease categories. The density of the conditional distribution of all SCS, given $\mathbf{Z}=\mathbf{z}$ and $\alpha$ is

$$
\begin{gathered}
p(\mathbf{S C S} \mid \boldsymbol{\alpha}, \mathbf{Z}=\mathbf{z})=\prod_{i=1}^{n}\left[N\left(f_{i}(\boldsymbol{\alpha}), g_{i}(\boldsymbol{\alpha})\right) Z_{i}\right. \\
\left.+N^{*}\left(f_{i}^{*}(\boldsymbol{\alpha}), g_{i}^{*}(\boldsymbol{\alpha})\right)\left(1-Z_{i}\right)\right]= \\
\prod_{i=1}^{n}\left[N\left(f_{i}(\boldsymbol{\alpha}), g_{i}(\boldsymbol{\alpha})\right) I\left(\lambda_{i}>T\right)+N^{*}\left(f_{i}^{*}(\boldsymbol{\alpha}), g_{i}^{*}(\boldsymbol{\alpha})\right) I\left(\lambda_{i} \leq T\right)\right]
\end{gathered}
$$

where the indicator $I\left(\lambda_{i}>T\right)=1$ if $\lambda_{i}>T$, and 0 elsewhere, and $I\left(\lambda_{i} \leq T\right)=1$ if $\lambda_{i} \leq T$ and 0 elsewhere. It is assumed that

$$
\begin{aligned}
& f_{i}^{*}(\boldsymbol{\alpha})=\mathbf{x}_{\mathbf{i} \mathbf{0}_{\mathbf{S C S}}}^{\prime} \boldsymbol{\beta}_{\mathbf{S C S}}+\mathbf{w}_{\mathbf{i a}}^{\prime} \mathbf{a}_{\mathbf{S C S}}+\mathbf{w}_{\mathbf{i p}}^{\prime} \mathbf{p}_{\mathbf{S C S}},
\end{aligned}
$$

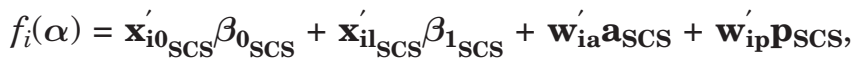

$$
\begin{aligned}
& g_{i}^{*}(\boldsymbol{\alpha})=\sigma_{\mathrm{e}{ }_{S C S}}^{2} \text { and } g_{i}(\boldsymbol{\alpha})=\sigma_{\mathrm{e}_{S C S}}^{2},
\end{aligned}
$$

where $\mathbf{x}^{\prime}$ and $\mathbf{w}^{\prime}$ with appropriate subscripts are incidence row vectors. Further, it is assumed that the $\lambda_{i}$, given $\alpha$, are mutually independent so that the density of the $\lambda$ vector, given $\alpha$, is

$$
p(\boldsymbol{\lambda} \mid \boldsymbol{\alpha})=\prod_{i=1}^{n} p\left(\lambda_{i} \mid \boldsymbol{\alpha}\right),
$$

where the distribution of $\lambda_{i}$, given $\alpha$, is

$$
\lambda_{i} \mid \boldsymbol{\alpha} \sim N\left(\mathbf{x}_{\mathbf{i}_{\lambda}}^{\prime} \boldsymbol{\beta}_{\lambda}+\mathbf{w}_{\mathbf{i} \mathbf{a}}^{\prime} \mathbf{a}_{\lambda}+\mathbf{w}_{\mathbf{i} \mathbf{p}}^{\prime} \mathbf{p}_{\lambda}, \sigma_{e_{\lambda}}^{2}\right) .
$$

For reasons of identifiability, it will be assumed that $\sigma_{e_{\lambda}}^{2}=1$. With this structure, [4] is equivalent to

$$
P_{i}=\Phi\left(\mathbf{x}_{\mathbf{i}_{\lambda}}^{\prime} \boldsymbol{\beta}_{\lambda}+\mathbf{w}_{\mathbf{i} \mathbf{a}}^{\prime} \mathbf{a}_{\lambda}+\mathbf{w}_{\mathbf{i}}^{\prime} \mathbf{p}_{\lambda}\right)=\Phi\left(\tilde{\lambda}_{i}\right)
$$

where $\Phi(\cdot)$ is the standard normal cumulative distribution function and $\tilde{\lambda}_{i}$ is the expectation of the liability of observation $i$, conditionally on $\boldsymbol{\beta}_{\lambda}, \mathbf{a}_{\lambda}$, and $\mathbf{p}_{\lambda}$. Thus, in our extended model, $P_{i}$ is a function of location parameters affecting liability (included in $\boldsymbol{\alpha}$ ), which may differ between observations.

\section{Bayesian Structure}

Conditional density of SCS. The conditional distribution of the data given the parameters $(\mathbf{P}, \boldsymbol{\alpha})$ is $p(\mathbf{S C S} \mid \mathbf{P}, \boldsymbol{\alpha})$ as in [2]. Further, the conditional distribution of SCS, given $\boldsymbol{\alpha}$ and $\mathbf{Z}$, is given in [6]. Also, from [5], $p(\mathbf{S C S} \mid \boldsymbol{\alpha}, \boldsymbol{\lambda}, \mathbf{Z}=\mathbf{z})=p(\mathbf{S C S} \mid \boldsymbol{\alpha}, \mathbf{Z}=\mathbf{z})$.

Prior density of all unknown parameters. The joint prior density of all unknown parameters, including the liabilities $(\boldsymbol{\lambda})$ as unknowns, is 


$$
\begin{gathered}
p(\boldsymbol{\alpha}, \boldsymbol{\lambda}, \mathbf{Z}=\mathbf{z})=\operatorname{Pr}(\mathbf{Z}=\mathbf{z} \mid \boldsymbol{\alpha}, \boldsymbol{\lambda}) p(\boldsymbol{\lambda} \mid \boldsymbol{\alpha}) p(\boldsymbol{\alpha})=[9] \\
\operatorname{Pr}(\mathbf{Z}=\mathbf{z} \mid \boldsymbol{\lambda}) p(\boldsymbol{\lambda} \mid \boldsymbol{\alpha}) p(\boldsymbol{\alpha}) .
\end{gathered}
$$

Note that $\operatorname{Pr}\left(Z_{i}=0 \mid \lambda_{i}\right)=\left\{\begin{array}{l}1 \text { if } \lambda_{i} \leq T \\ 0 \text { if } \lambda_{i}>T\end{array}\right.$, and

$\operatorname{Pr}\left(Z_{i}=1 \mid \lambda_{i}\right)=\left\{\begin{array}{l}0 \text { if } \lambda_{i} \leq T \\ 1 \text { if } \lambda_{i}>T\end{array}\right.$. Hence, given $\lambda, \mathbf{Z}$ is com-

pletely specified, and $\operatorname{Pr}(\mathbf{Z}=\mathbf{z} \mid \boldsymbol{\lambda})$ is, therefore, a degenerate distribution. The density $p(\boldsymbol{\lambda} \mid \boldsymbol{\alpha})$ is defined in [7], and the density $p(\boldsymbol{\alpha})$ is

$$
\begin{gathered}
p(\boldsymbol{\alpha})= \\
p(\boldsymbol{\beta}) p\left(\mathbf{a} \mid \mathbf{G}_{\mathbf{0}}\right) p\left(\mathbf{p} \mid \mathbf{P}_{\mathbf{0}}\right) p\left(\mathbf{G}_{\mathbf{0}}\right) p\left(\mathbf{P}_{\mathbf{0}}\right) p\left(\sigma_{\mathrm{e}{ }_{\mathbf{S C S}}^{2}}^{2}\right) p\left(\sigma_{{ }_{\mathrm{e} 1}}^{2}\right)
\end{gathered}
$$

where $\mathrm{p}(\boldsymbol{\beta}), \mathrm{p}\left(\mathbf{G}_{0}\right), \mathrm{p}\left(\mathbf{P}_{0}\right), \mathrm{p}\left(\sigma_{\mathrm{e} 0_{\mathrm{SCS}}}^{2}\right), \mathrm{p}\left(\sigma_{\mathrm{e}{ }_{\mathrm{SCS}}}^{2}\right)$ were assigned bounded uniform priors. To achieve reasonably vague priors, the absolute values of the bounds were large. Further, to avoid "label-switching" problems (McLachlan and Peel, 2000), constraints must be imposed on parameters of the SCS distributions of putative IMI- and IMI+ animals; for example, the mean SCS in the IMI- group was set to be lower than that in the IMI+ group.

Additive breeding values (a) and permanent environmental effects (p) were assumed to be normally distributed, with the densities

$$
\mathrm{p}\left(\mathbf{a} \mid \mathbf{G}_{\mathbf{0}}\right) \propto\left|\mathbf{G}_{\mathbf{0}}\right|^{-\frac{q_{a}}{2}} \exp \left\{-\frac{1}{2} \mathbf{a}^{\prime}\left(\mathbf{G}_{\mathbf{0}}^{-\mathbf{1}} \otimes \mathbf{A}^{-\mathbf{1}}\right) \mathbf{a}\right\}
$$

and

$$
\mathrm{p}\left(\mathbf{p} \mid \mathbf{P}_{\mathbf{0}}\right) \propto\left|\mathbf{P}_{\mathbf{0}}\right|^{-\frac{q_{p}}{2}} \exp \left\{-\frac{1}{2} \mathbf{p}^{\prime}\left(\mathbf{P}_{\mathbf{0}}^{-\mathbf{1}} \otimes \mathbf{I}\right) \mathbf{p}\right\}
$$

where $\mathbf{A}$ is the additive relationship matrix with dimen$\operatorname{sion} q_{a}$, I is an identity matrix with dimension $q_{p}$ (number of individuals with at least one SCS record), and $\mathbf{G}_{\mathbf{0}}$ and $\mathbf{P}_{\mathbf{0}}$ are as previously defined.

Joint posterior density. The augmented joint posterior density of all unknowns is

$$
\begin{gathered}
p(\boldsymbol{\alpha}, \boldsymbol{\lambda}, \mathbf{Z}=\mathbf{z} \mid \mathbf{S C S}) \\
\propto p(\mathbf{S C S} \mid \boldsymbol{\alpha}, \boldsymbol{\lambda}, \mathbf{Z}=\mathbf{z}) p(\boldsymbol{\alpha}, \boldsymbol{\lambda}, \mathbf{Z}=\mathbf{z}) \\
\propto p(\mathbf{S C S} \mid \boldsymbol{\alpha}, \mathbf{Z}=\mathbf{z}) \operatorname{Pr}(\mathbf{Z}=\mathbf{z} \mid \boldsymbol{\lambda}) p(\boldsymbol{\lambda} \mid \boldsymbol{\alpha}) p(\boldsymbol{\beta}) \\
\mathrm{p}\left(\mathbf{a} \mid \mathbf{G}_{\mathbf{0}}\right) p\left(\mathbf{p} \mid \mathbf{P}_{\mathbf{0}}\right) p\left(\mathbf{G}_{\mathbf{0}}\right) p\left(\mathbf{P}_{\mathbf{0}}\right) p\left(\sigma_{e 0_{S C S}}^{2}\right) p\left(\sigma_{e 1_{S C S}}^{2}\right)
\end{gathered}
$$

Fully conditional posterior distributions. Knowledge of the fully conditional posterior distribution of each parameter (or block of parameters) is required for implementing a Gibbs sampler. Given $\mathbf{Z}$ and $\lambda$, all fully conditional posterior distributions have a standard form, and, therefore, are easy to sample from (Sorensen and Gianola, 2002). Specifically, given $\mathbf{Z}$, the required posterior distributions are as in a linear Gaussian model: 1) the conditional posterior distribution of each element of $\beta$ is normal, truncated in some interval $\left[d_{1}, d_{2}\right] ; 2$ ) the conditional distributions of both $\mathbf{a}$ and $\mathbf{p}$ are multivariate normal; 3 ) the densities of $\mathbf{G}_{\mathbf{0}}$ and $\mathbf{P}_{\mathbf{0}}$ are inverse Wishart, and 4) the conditional distributions of $\sigma_{\mathrm{e} 0}^{2}$ and $\sigma_{\mathrm{e}_{S C S}}^{2}$ are inverse gamma.

Further one has

$$
\begin{gathered}
p(\lambda, \mathbf{Z}=\mathbf{z} \mid \alpha, \mathbf{S C S})= \\
\operatorname{Pr}(\mathbf{Z}=\mathbf{z} \mid \alpha, \mathbf{S C S}) p(\boldsymbol{\lambda} \mid \boldsymbol{\alpha}, \mathbf{Z}=\mathbf{z}, \mathbf{S C S})
\end{gathered}
$$

with $p(\boldsymbol{\lambda} \mid \boldsymbol{\alpha}, \mathbf{Z}=\mathbf{z}, \mathbf{S C S})=p(\boldsymbol{\lambda} \mid \boldsymbol{\alpha}, \mathbf{Z}=\mathbf{z})$, because the liabilities are independent of SCS, given $\alpha$ and $\mathbf{Z}=\mathbf{z}$. Now, the probability

$$
\operatorname{Pr}(\mathbf{Z}=\mathbf{z} \mid \boldsymbol{\alpha}, \mathbf{S C S})=\prod_{i=1}^{n} \operatorname{Pr}\left(Z_{i}=z_{i} \mid \boldsymbol{\alpha}, S C S_{i}\right)
$$

is a product of $n$ independent Bernoulli distributions with success probability $\tau_{i}$. As $Z_{i}=1$ is equivalent to $\lambda_{i}$ $>0$ :

$$
\begin{gathered}
\operatorname{Pr}\left(Z_{i}=1 \mid \alpha, S C S_{i}\right) \\
\propto p\left(S C S_{i} \mid \alpha, \lambda_{i}>0\right) \int_{0}^{\infty} p\left(\lambda_{i} \mid \boldsymbol{\alpha}\right) d \lambda_{i} \\
\propto p\left(S C S_{i} \mid \alpha, Z_{i}=1\right) \Phi\left(\tilde{\lambda}_{i}\right) ;
\end{gathered}
$$

likewise

$$
\begin{gathered}
\operatorname{Pr}\left(Z_{i}=0 \mid \alpha, S C S_{i}\right) \\
\propto p\left(S C S_{i} \mid \alpha, \lambda_{i} \leq 0\right) \int_{-\infty}^{0} p\left(\lambda_{i} \mid \alpha\right) d \lambda_{i} \\
\propto p\left(S C S_{i} \mid \alpha, Z_{i}=0\right)\left(1-\Phi\left(\tilde{\lambda}_{i}\right)\right) .
\end{gathered}
$$

The parameter $\left(\tau_{i}\right)$ of the fully conditional posterior distribution of $Z_{i}$ (Bernoulli) is therefore

$$
\begin{gathered}
\tau_{i}= \\
\frac{p\left(S C S_{i} \mid \boldsymbol{\alpha}, Z_{i}=1\right) \Phi\left(\tilde{\lambda}_{i}\right)}{p\left(S C S_{i} \mid \boldsymbol{\alpha}, Z_{i}=0\right)\left(1-\Phi\left(\tilde{\lambda}_{i}\right)\right)+p\left(S C S_{i} \mid \boldsymbol{\alpha}, Z_{i}=1\right) \Phi\left(\tilde{\lambda}_{i}\right)}
\end{gathered}
$$


Hence, $Z_{i}$ can be sampled from a Bernoulli distribution with probability [17]. Subsequently, given $Z_{i}, \lambda_{i}$ can be sampled from a distribution with density

$$
\begin{gathered}
p\left(\lambda_{i} \mid \alpha, Z_{i}=z_{i}, S C S_{i}\right)=p\left(\lambda_{i} \mid \alpha, Z_{i}=z_{i}\right) \\
\propto p\left(S C S_{i} \mid \alpha, Z_{i}=z_{i}\right) p\left(\lambda_{i} \mid \alpha, Z_{i}=z_{i}\right) .
\end{gathered}
$$

Given $Z_{i}$, the term $p\left(S C S_{i} \mid \alpha, Z_{i}=z_{i}\right)$ does not involve $\lambda_{i}$ and is thus a constant. Hence,

$$
\begin{gathered}
p\left(\lambda_{i} \mid \alpha, Z_{i}=z_{i}\right)= \\
\left(1-Z_{i}\right) p\left(\lambda_{i} \mid \alpha, \lambda_{i} \leq 0\right)+Z_{i} p\left(\lambda_{i} \mid \alpha, \lambda_{i}>0\right) .
\end{gathered}
$$

In other words, the fully conditional distribution of $\lambda_{i}$, given $Z_{i}$, is a truncated standard normal distribution $\operatorname{TN}\left(\lambda_{\mathrm{i}} \mid \alpha\right)$, with right truncation for $Z_{i}=0$ and left truncation for $Z_{i}=1$.

\section{Implementation of a Gibbs Sampler}

The following steps describe how Gibbs sampling can be conducted for the LNM model.

1. Set initial values for parameters as needed. Set initial values for $Z_{i}$, for $i=1,2, \ldots, n$. (e.g, $Z_{i}=1$ for observed SCS above a certain threshold, and $Z_{i}=0$ otherwise).

2. Sample $\lambda_{i}$ as described in [19], for $i=1,2, \ldots, n$.

3. Sample all parameters in $\alpha$ as for a standard Gaussian bivariate model (for SCS and $\lambda$ ), assuming a zero residual covariance between the traits.

4. Compute $\tau_{i}$, as described in [17], and sample $Z_{i}$ from a Bernoulli distribution with parameter $\tau_{i}$ for $i=1,2, \ldots, n$.

5. Repeat ( 2 through 4 ) as many times as needed for burn-in and desired level of Monte Carlo error.

\section{Simulation Study}

Four different scenarios were simulated. Input parameters are given in Table 1 . In all settings, SCS was assumed to follow a 2-component mixture, which depended on a putative mastitis status, determined fully by an underlying liability. Both SCS and liability to mastitis were simulated with additive genetic and permanent environmental effects in addition to a random residual following standard procedures. The difference between means of the 2 normal distributions in the SCS mixture equaled 2 units for all scenarios, and residual variance for SCS was assumed homogeneous. Four generations, each consisting of 800 cows, progeny of 10 sires, and 10 records per cow, were simulated. No selection was applied, sires were recruited from 10 different bull dams, and all sires in each generation had the same probability of siring offspring of both sexes. Each cow was replaced by a daughter, and mating was at random. Inbreeding was ignored. Intended mastitis frequency was set to $25 \%$.

Different biological relationships between SCS and mastitis were assumed for the 4 scenarios (Table 1 ). In scenarios 1 and 4 , the genetic correlation between baseline SCS and liability was set to zero; it was assumed positive (0.5) in scenario 2 and negative $(-0.5)$ in scenario 3. A positive genetic correlation indicates that cows with a genetically high baseline SCS are more susceptible to infection; the opposite is true when assuming a negative correlation. In the first 3 scenarios, heritability of both SCS and liability was assumed 0.1; in scenario 4 , heritability of liability was set equal to 0.05 , while heritability of SCS was kept at 0.1 . In all scenarios, permanent environmental variance for both baseline SCS and liability was assumed to be $10 \%$ of the total variance of the trait. Each scenario was replicated 20 times.

The following LNM model was used in the genetic analyses, given the vector of indicator variables $\mathbf{z}$ :

$$
\left[\begin{array}{c}
\mathbf{S C S} \\
\lambda
\end{array}\right]=\left[\begin{array}{c}
\mathbf{1} \mu_{S C S}+\mathbf{z} \Delta+\mathbf{W}_{\mathbf{a}} \mathbf{a}_{S C S}+\mathbf{W}_{\mathbf{p}} \mathbf{p}_{S C S}+\mathbf{e}_{S C S} \\
\mathbf{1} \mu_{\lambda}+\mathbf{W}_{\mathbf{a}} \mathbf{a}_{\lambda}+\mathbf{W}_{\mathbf{p}} \mathbf{p}_{\lambda}+\mathbf{e}_{\lambda}
\end{array}\right]
$$

where $\mu_{S C S}$ and $\mu_{\lambda}$ are mean values of SCS and liability, respectively, and $\Delta$ is the difference between means of the 2 components in the mixture (effect of mastitis on SCS). For comparison purposes, a mixture model ignoring the structure of the underlying liability to mastitis (NM) (Ødegård et al., 2003) was also fitted:

$$
\mathbf{S C S}=\mathbf{1} \mu_{S C S}+\mathbf{z} \Delta+\mathbf{W}_{\mathbf{a}} \mathbf{a}_{S C S}+\mathbf{W}_{\mathbf{p}} \mathbf{p}_{S C S}+\mathbf{e}_{S C S} .
$$

The following standard univariate model for SCS, ignoring the mixture (IM) was fitted as well

$$
\mathbf{S C S}=\mathbf{1} \mu_{S C S}+\mathbf{W}_{\mathbf{a}} \mathbf{a}_{S C S}+\mathbf{W}_{\mathbf{p}} \mathbf{p}_{S C S}+\mathbf{e}_{S C S} .
$$

In the LNM and NM models, features of the posterior distributions were estimated with Gibbs sampling. A chain consisting of 10,000 burn-in rounds and 100,000 sampling rounds was run. In the IM model, parameters were estimated with the AI-REML algorithm. All computations were carried out using a modified version of the DMU package (Madsen and Jensen, 2005).

The 2 mixture models (LNM vs. NM) were compared on the basis of their ability to classify SCS observation into correct disease categories, defined as sensitivity (average posterior probability of IMI+ for truly diseased animals) and specificity (average posterior probability of IMI- for truly healthy animals), ability to return 
Table 1. Input parameters (IP) and posterior means (PM) from a liability normal mixture (LNM) model and a normal mixture model (NM) under four different scenarios. Posterior means are presented as averages over 20 replicated simulations, with corresponding between-replicate standard deviations of posterior means (SD).

\begin{tabular}{|c|c|c|c|c|c|c|c|c|c|}
\hline \multirow[b]{2}{*}{ Scenario } & \multirow[b]{2}{*}{ Model } & \multicolumn{8}{|c|}{ Parameter $^{1}$} \\
\hline & & & $\sigma_{\mathrm{a}_{\mathrm{SCS}}}^{2}$ & $\sigma_{\mathrm{a}_{\lambda}}^{2}$ & $\mathrm{r}_{\mathrm{a}_{\mathrm{SCS}, \lambda}}$ & $\sigma_{\mathrm{p}_{\mathrm{SCS}}}^{2}$ & $\sigma_{\mathrm{p}_{\lambda}}^{2}$ & $\mathrm{r}_{\mathrm{p}_{\mathrm{SCS}, \lambda}}$ & $\sigma_{\mathrm{e}_{\mathrm{SCS}}}^{2}$ \\
\hline \multirow[t]{5}{*}{1} & \multirow{3}{*}{ LNM } & IP & 0.100 & 0.125 & 0.000 & 0.100 & 0.125 & 0.000 & 0.800 \\
\hline & & $\mathrm{PM}$ & 0.095 & 0.117 & 0.060 & 0.102 & 0.119 & 0.029 & 0.798 \\
\hline & & SD & 0.015 & 0.031 & 0.201 & 0.016 & 0.031 & 0.148 & 0.014 \\
\hline & \multirow[t]{2}{*}{$\mathrm{NM}$} & $\mathrm{PM}$ & 0.125 & - & - & 0.128 & - & - & 0.814 \\
\hline & & $\mathrm{SD}$ & 0.016 & - & - & 0.013 & - & - & 0.017 \\
\hline \multirow[t]{5}{*}{2} & \multirow{3}{*}{ LNM } & IP & 0.100 & 0.125 & 0.500 & 0.100 & 0.125 & 0.000 & 0.800 \\
\hline & & $\mathrm{PM}$ & 0.099 & 0.116 & 0.545 & 0.098 & 0.126 & 0.093 & 0.803 \\
\hline & & SD & 0.010 & 0.020 & 0.136 & 0.012 & 0.033 & 0.126 & 0.012 \\
\hline & \multirow{2}{*}{$\mathrm{NM}$} & PM & 0.172 & - & - & 0.133 & - & - & 0.806 \\
\hline & & $\mathrm{SD}$ & 0.020 & - & - & 0.013 & - & - & 0.014 \\
\hline \multirow[t]{5}{*}{3} & \multirow{3}{*}{ LNM } & IP & 0.100 & 0.125 & -0.500 & 0.100 & 0.125 & 0.000 & 0.800 \\
\hline & & $\mathrm{PM}$ & 0.092 & 0.115 & -0.481 & 0.104 & 0.119 & 0.083 & 0.798 \\
\hline & & SD & 0.022 & 0.036 & 0.155 & 0.015 & 0.036 & 0.152 & 0.018 \\
\hline & \multirow{2}{*}{ NM } & PM & 0.070 & - & - & 0.136 & - & - & 0.823 \\
\hline & & $\mathrm{SD}$ & 0.013 & - & - & 0.015 & - & - & 0.016 \\
\hline \multirow[t]{5}{*}{4} & \multirow{3}{*}{ LNM } & IP & 0.100 & 0.059 & 0.000 & 0.100 & 0.118 & 0.000 & 0.800 \\
\hline & & $\mathrm{PM}$ & 0.105 & 0.077 & 0.012 & 0.094 & 0.134 & 0.094 & 0.800 \\
\hline & & SD & 0.018 & 0.019 & 0.202 & 0.016 & 0.033 & 0.189 & 0.013 \\
\hline & \multirow[t]{2}{*}{$\mathrm{NM}$} & PM & 0.117 & - & - & 0.127 & - & - & 0.810 \\
\hline & & SD & 0.016 & - & - & 0.015 & - & - & 0.012 \\
\hline
\end{tabular}

${ }^{1} \sigma_{\mathrm{a}_{\mathrm{SCS}}}^{2}=$ Additive genetic variance for SCS, $\sigma_{\mathrm{a}_{\lambda}}^{2}=$ additive genetic variance for liability to putative mastitis, $\mathrm{r}_{\mathrm{a}_{\mathrm{SCS}, \lambda}}=$ additive genetic correlation between SCS and liability to putative mastitis, $\sigma_{\mathrm{P}_{\mathrm{SCS}}}^{2}=$ permanent environmental variance for SCS, $\sigma_{\mathrm{p}_{\lambda}}^{2}=$ permanent environmental variance for liability to putative mastitis, $\mathrm{r}_{\mathrm{p}_{\mathrm{SCS}, \lambda}}=$ correlation between permanent environmental effects for SCS and liability to putative mastitis, and $\sigma_{\mathrm{e}_{\mathrm{SCS}}}^{2}=$ residual variance for SCS.

simulated parameter values (LNM vs. NM), and accuracy of selection (LNM vs. IM). Accuracy of selection was defined as the correlation between the selection criterion and the true breeding value for liability to mastitis.

\section{RESULTS AND DISCUSSION}

\section{Simulation Study}

Across-replicate averages of posterior means and standard deviations of posterior means estimated with the LNM and NM models under the 4 scenarios are presented in Table 1. Use of the LNM model (used to generate the data) yielded parameter estimates that were close to the input parameters. However, if heterogeneity in the underlying liability to mastitis was ignored in the mixture model fitted, as in the NM model, all variance components were systematically overestimated, with additive genetic variance in scenario 3 as the only exception. The bias could be explained by a confounding between parameters of the liability distri- bution with those of the baseline SCS. This means that variation in mastitis liability may be partially misinterpreted as variation in baseline SCS (e.g., cows with a high prior probability of mastitis may be viewed as having a high baseline SCS). In scenario 3, a negative genetic correlation between baseline SCS and liability was assumed. Here, families with a low baseline SCS also have a higher risk of infection and, therefore, more frequent SCS elevations, and vice versa. This may reduce variation in observed SCS between families and, thus, explain why additive genetic variance was somewhat underestimated using the NM model for this scenario.

When using a LNM model, sensitivity (probability of classifying a diseased animal as diseased) and specificity (probability of classifying a healthy animal as healthy) were slightly increased relative to the NM model (Table 2). In the LNM model, sensitivity ranged from 0.645 to 0.664 , and specificity varied between 0.881 and 0.893 . Because we assumed a frequency of mastitis of 0.25 , the probability of an error of status 
Table 2. Sensitivity and specificity estimates ${ }^{1}$ for the 4 scenarios from a liability normal mixture model (LNM) and a normal mixture model (NM). Estimates are means of posterior means for 20 replicates for each scenario.

\begin{tabular}{llllll}
\hline & \multicolumn{2}{c}{ LNM } & & \multicolumn{2}{c}{ NM } \\
\cline { 2 - 3 } \cline { 5 - 6 } Scenario & Sensitivity & Specificity & & Sensitivity & Specificity \\
\hline 1 & 0.660 & 0.886 & & 0.626 & 0.881 \\
2 & 0.664 & 0.893 & & 0.624 & 0.886 \\
3 & 0.648 & 0.881 & & 0.618 & 0.877 \\
4 & 0.645 & 0.892 & & 0.617 & 0.889 \\
\hline
\end{tabular}

${ }^{1}$ Sensitivity and specificity are defined as probability of correct classification of observations from diseased and healthy animals, respectively.

classification is given by $[0.25 \times(1-$ sensitivity $)+0.75$ $\times(1-$ specificity $)]$. The implication for the best-case scenario (the model fitting perfectly, as in the LNM model) is that the expected frequency of errors of classification would be around $0.25 \times 0.34+0.75 \times 0.11=$ 0.17 . When more complicated explanatory structures (e.g., herd-specific effects, age at calving, and stage of lactation) are needed to describe the data, modeling correctly the underlying liability to mastitis might be more crucial, because such factors may have rather different effects with respect to baseline SCS and liability to mastitis.

If the LNM model holds, posterior means of predicted breeding values for liability to mastitis from the LNM model would be the preferred selection criteria, whereas in a standard linear model for SCS (IM), selection criteria are simply predicted breeding values for SCS. Figure 1 shows that there was a substantial, but highly variable, increase in accuracy of selection by using a

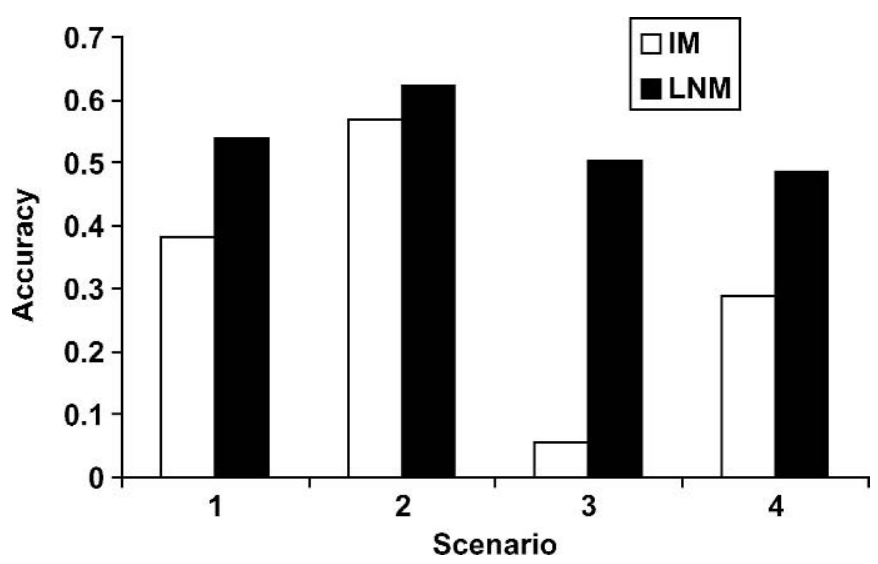

Figure 1. Accuracy of selection measured as correlation between true breeding values for liability to mastitis and selection criteria using either a standard linear model for SCS ignoring the mixture (IM) (EBV for SCS) or a liability normal mixture model (LNM) (EBV for liability to mastitis). The correlations are averaged over 20 replicates for each scenario.
LNM model from that attained with the standard linear model for SCS (IM) used routinely in genetic evaluation for the purposes of indirect selection against mastitis. The increase was largest in scenario 3 , where the genetic correlation between baseline SCS and liability was negative, and lowest when assuming a positive genetic correlation (scenario 2). When using the IM model, selection would be for a lower SCS. This is expected to reduce incidence of mastitis and to lower baseline SCS. If the genetic correlation between baseline SCS and liability is positive, selection for lower baseline will also reduce incidence of mastitis. However, the opposite would be true if the genetic correlation is negative. This explains the large differences in the superiority of the LNM relative to the IM model in different scenarios.

\section{Future Developments}

Given the promise shown in simulations by the NM model and by the proposed LNM model over the standard linear specification used in analysis of SCS, it is critical to carry out applications with real data. For example, using the Norwegian health database, it would be possible to evaluate the behavior of the alternative models in practice, as true clinical mastitis status is recorded on a large scale.

The proposed LNM model could be extended to multivariate mixtures, i.e., several mixture traits depending on the same status variable (e.g., SCS and electrical conductivity in milk) or on different indicator variables (e.g., SCS and a trait affected by a QTL). Similarly, it is of interest to develop models for joint analysis of mixture and non-mixture traits (e.g., SCS and longevity). More advanced mixtures consisting of $>2$ components (e.g., mastitis caused by different pathogens) may be developed as well.

In addition to selecting cows that are able to avoid infection, it is also desirable to improve, genetically, the cows' ability to recover from infection. This may be achieved by developing a mixture model where the magnitude of the SCS response to infection has a genetic component, which, in turn, may be related to the probability of recovery from disease.

\section{CONCLUSION}

Based on simulations, it was illustrated that the proposed liability normal mixture model yielded seemingly unbiased estimates of all simulated parameters and that it has the potential of increasing accuracy of genetic evaluation relative to a standard linear model. The proposed model can be extended to handle a wider range of problems related to genetic analyses in which a finite mixture specification is warranted. 


\section{ACKNOWLEDGMENTS}

The project has received funding from The Nordic Joint Committee for Agricultural Research (NKJ) and The Nordic Academy for Advanced Study (NorFA). D. Gianola's contribution to this research was partly funded by grants NRICGP/USDA 2003-35205-12833 and NSF DEB-0089742.

\section{REFERENCES}

Chang, Y. M., D. Gianola, B. Heringstad, and G. Klemetsdal. 2004. Longitudinal analysis of clinical mastitis at different stages of lactation in Norwegian Cattle. Livest. Prod. Sci. 88:251-261.

Dempster, E. R., and I. M. Lerner. 1950. Heritability of threshold characters. Genetics 35:212-235.

Falconer, D. S. 1965. The inheritance of liability to certain diseases estimated from the incidence among the relatives. Ann. Hum. Genet. 29:51-76.

Gianola, D. 1982. Theory and analysis of threshold characters. J. Anim. Sci. 54:1079-1096.

Gianola, D., and J. L. Foulley. 1983. Sire evaluation for ordered categorical data with a threshold model. Genet. Sel. Evol. 15:201-223.
Gianola, D., J. Ødegård, B. Heringstad, G. Klemetsdal, D. Sorensen, P. Madsen, J. Jensen, and J. Detilleux. 2004. Mixture model for inferring susceptibility to mastitis in dairy cattle: A procedure for likelihood-based inference. Genet. Sel. Evol. 36:3-27.

Heringstad, B., G. Klemetsdal, and J. Ruane. 1999. Clinical mastitis in Norwegian Cattle: Frequency, variance components, and genetic correlation with protein yield. J. Dairy Sci. 82:1325-1330.

Heringstad, B., R. Rekaya, D. Gianola, G. Klemetsdal, and K. A. Weigel. 2003. Genetic change for clinical mastitis in Norwegian Cattle: A threshold model analysis. J. Dairy Sci. 86:369-375.

Madsen, P., and J. Jensen. 2005. A User's Guide to DMU. A Package for Analyzing Multivariate Mixed Models. Version 6, release 4.5. Danish Institute of Agricultural Sciences, Research Centre Foulum, Tjele, Denmark.

McLachlan, G., and D. Peel. 2000. Finite Mixture Models. John Wiley \& Sons, Inc., New York, NY.

Ødegård, J., J. Jensen, P. Madsen, D. Gianola, G. Klemetsdal, and B. Heringstad. 2003. Detection of mastitis in dairy cattle by use of mixture models for repeated somatic cell scores: A Bayesian approach via Gibbs sampling. J. Dairy Sci. 86:3694-3703.

Sorensen, D., and D. Gianola. 2002. Likelihood, Bayesian, and MCMC Methods in Quantitative Genetics. Springer-Verlag, New York, NY.

Wright, S. 1934. An analysis of variability in number of digits in an inbred strain of Guinea pigs. Genetics 19:506-536. 